\begin{tabular}{ccc}
\hline International Journal of Engineering \&Technology, $7(3.12)(2018) 565-567$ \\
SPC & Website: www.sciencepubco.com/index.php/IJET \\
Research paper & International Journal of Engineering \& Technology \\
\hline
\end{tabular}

\title{
Efficient Attendance Management System Based on Facial Recognition
}

\author{
SetuChoudhary $^{1 *}$, AayushKakaji ${ }^{2}$, Kusuma Pranay $^{3}$, P.Prabhu $^{4}$ \\ Department of Electronics and Communication Engineering, SRM Institute of Science and Technology, \\ Kanchipuram, India
}

\begin{abstract}
A Face recognition system is an application of computer vision which is capable of performing two major tasks identifying and verifying a person from given data base. The objective of this paper is to design an effective attendance system which is based on facial recognition and intend to reduce the manual efforts of the teacher. In the conventional attendance system there are several issue like fake attendance, time consumption, manipulation of attendance. The algorithm used is named fisher face algorithm, which is already in use but it gives an accuracy of 5-6\% and the amount of faces it can detect is comparatively less, Here we intend to use fisher face algorithm with the help of support vector machine(SVM). The system is trained with database faces. The data gets updated in the portal which is accessed by the faculty and the students. This paper is a speculative model of attendance management system using facial recognition.
\end{abstract}

\section{Introduction}

Automated Face recognition finds major application in the field of education to expeditiously automate and carry off the attendance system. As the number of students enrolling to university increases every year, to maintain the attendance and records of thousands of students is a major concern in the education sector. Therefore we discuss an effective system to mark attendance of students automatically by recognizing their face.

\section{Pre-Existing Methodologies}

Initially the technology was based on finger print sensor [1],in this system there were a lot of problems that were being faced in the crowded places, as the number of devices were limited and number of people were more. so, many a times attendance mismatch occurred. It further extended to wireless system [3][4]. This system continued to hold limitations. The next development was providing RFID [5]. The concept was simple to provide a chip inside an ID card, but the system required large number of components and space. Though the chance of error in this system was very less, but the equipment cost was high and the maintenance was difficult. Further speech recognition system, was identified in this system the speech was taken and the attendance was recorded[2]. The system is bit complicated as there was no assurance thing, anyone could mark for anyone by recording their voice on the phone and playing it, so the system was very unreliable.

Further many papers were done for attendance using facial recognition.Drawbacks were that it was light dependent, did not work in ambient conditions, the accuracy was below 5\%.The system which is proposed, is based on machine learning algorithms, in this the faces are trained and the functionality of camera is made more better with the usage. The system is light independent and can have an accuracy of $60 \%$. Firstly the 3 distinct points of faces are highlighted this forms the basis of the data set, the three distinct faces are analyzed by Harr transform mechanism, and further the changes in them are detected [9][10]. Now when the face is recognized the database is set up in which there are 20 pictures captured to train the data. After this process is done the images are stored. The code is run and the camera starts, allowing the face to be detected. The faces start getting detected with the help of SVM and are matched with the database, permitting the attendance to get updated in the portal.

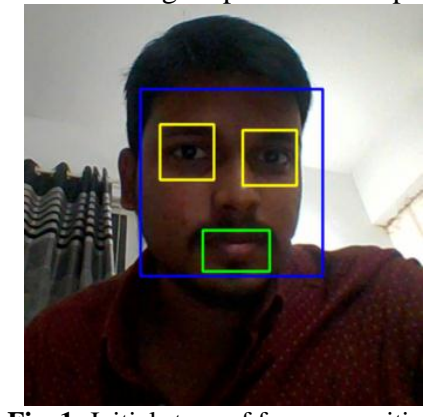

Fig. 1: Initial stage of face recognition

\section{Fisher Face Algorithm}

Fisher face algorithm conceives the ratio to the variation of one person and the other. It says that, this maximizes the determinant of between-class scatter matrix and minimize the determinant of within-class scatter matrix [6].The procedure of the Fisher face is as the following. Let the number of images be $\mathrm{N}$ and the total number of persons be c. For suppose, let $\mathrm{k}$ be the number of images from one person. PCA provides us with $\mathrm{N}-1$ ei-gen faces. For minimizing the determinant of within-class scatter matrix and maximizing the between-class scatter matrix, we represent the $S$, s', matrix and obtain Fisher faces.

$\mathrm{Sw}=\sum \mathrm{Cj}=1 \sum \mathrm{nji}=1(\mathrm{xij}-\mu \mathrm{j})(\mathrm{xij}-\mu \mathrm{j}) \mathrm{T}$, $\mathrm{Sb}=\sum \mathrm{Cj}=1(\mu \mathrm{j}-\mu)(\mu \mathrm{j}-\mu) \mathrm{T}$, 


\section{Proposed System Architecture}

A. Digital Image Acquisition

B. Face recognition

C. Face data-base generation

D. Automated attendance upload

\section{A. Digital Image Acquisition}

Images of students in the class are to be captured using a high definition web-camera which offer $1280 \times 720$ resolution. After capturing the images are stored in the data base.

\section{B. Face Recognition}

The camera detects the three portions of the face in separate frames. These portions which are detected are combined further and then form a single frame with the entire face detection and then furtherdisplays the name of the person.

\section{Face Data-Base Generation}

Initially the code is to be setup with the data of the students in the class and then the images are to be captured in accordance to the subject given in the code.For a given subject up to twenty images are to be captured. The captured images are tostored in the database.

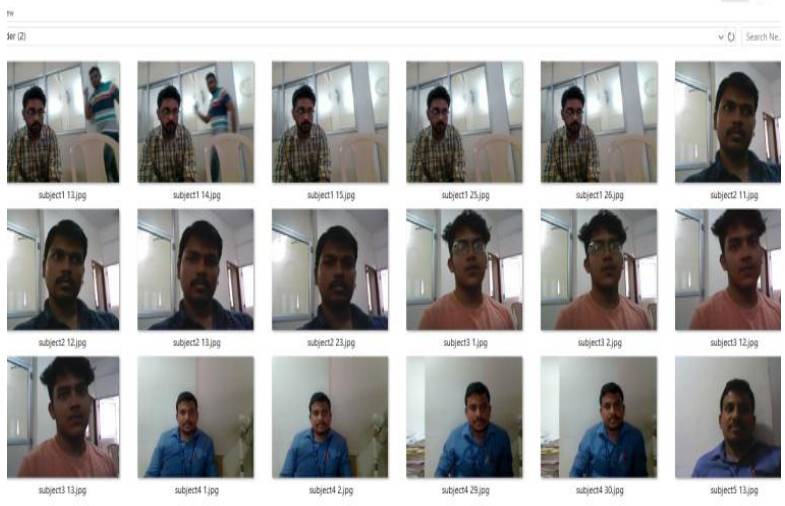

Fig. 2: Face Database

\section{Automated Attendance Upload}

After the detection of the faces the verification process is done,then the successful recognition of the faces is done. Then the attendance is automatically uploaded in the portal [11].

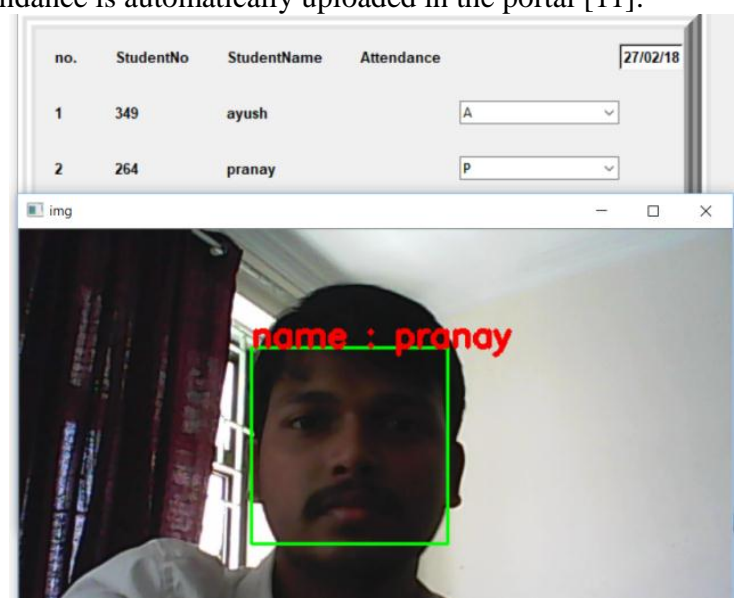

Fig. 3: Automated Attendance Portal

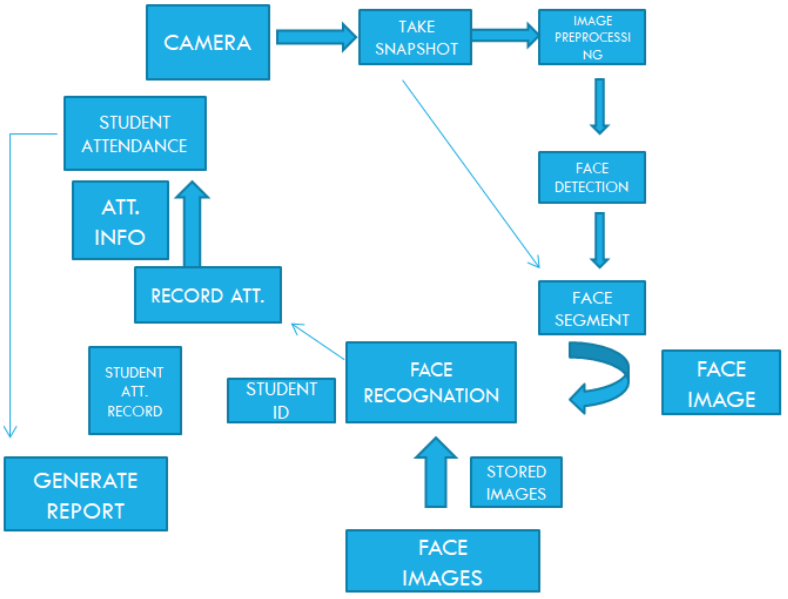

Fig. 4: Design flow

\section{Results and Discussion}

1. Initially the data base is loaded on running the python ide. And the images are converted into greyscale

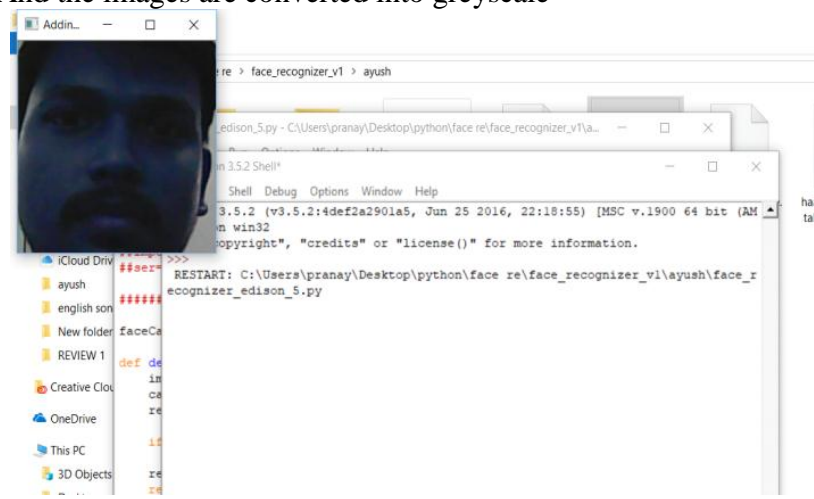

Fig. 5: Screen shot of grey scale image and code

2. Recognize the images stored in the data base, displays the name which includes a voice note.

3. Update the attendance portal-After face recognition, Attendance is automatically updated in the portal.

\section{Conclusions}

Apart from all Bio-metric techniques which exists, Automated Facial recognition technology has great expediency in the education field as it is used to update and manage the attendance automatically in a procure manner when compared to other traditional methods.In this paper, we consists a combination of face recognition and attendance upload portal which is explained using the Fisher face algorithm and obtained satisfactory results.

\section{References}

[1] S. Prabhakar, S. Pankanti, A.K. Jain, "Biometrics Recognition: Security and Privacy Concerns", IEEE Security \& Privacy, vol. 1, no. 2, pp. 33-42, 2003

[2] Pentland, B. Moghaddam, and T. Starner, "View-based and modular eigenspaces for face recognition," IEEE Conf. on Computer Vision and Pattern Recognition, MIT Media Laboratory Tech. Report No. 2451994

[3] NasliniK.Ratha, An FPGA-based Point Pattern Matching Processor with Application to Fingerprint Matching, the Computer Architectures for Machine Perception (CAMP '95). pp. 394401,1995

[4] B. Bhanu, X. Tan, "Learned templates for feature extraction in fingerprint images", Proceedings of the IEEE Conference on 
Computer Vision and Pettern recognition, vol.2, 2001, pp.591-596.

[5] T. Lim, S. Sim, and M. Mansor, "Rfid based attendance system", in Industrial Electronics \& Applications, 2009.ISIEA 2009.IEEE Symposium on, vol. 2. IEEE, 2009, pp. 778-782

[6] P.N. Belhumeour, J.P. Hespanha, and D.J. Kriegman, "Eigenfaces vs Fisherfaces: Recognition using class specific linear projection”, IEEE Transactions on Pattern Analysis and Machine Intelligence, 19(7):711-720, 1997

[7] Viola, P., Jones, M. Rapid Object Detection Using Boosted Cascade of Simple Features. IEEE Conference on Computer Vision and Pattern Recognition; 2001.

[8] Ming-Hsuan Yang, David J. Kriegman, Narendra Ahuja. Detecting Faces in Images: A Survey. IEEE Transactions on Pattern Analysis and Machine Intelligence.Vol. 24. NO 1.; 2002

[9] Yongsheng Gao; Leung, M.K.H., "Face recognition using line edge map", Pattern Analysis and Machine Intelligence, IEEE Transactions on, Volume: 24 Issue: 6 , June 2002, Page(s): 764 779.

[10] De Vel, O.; Aeberhard, S., "Line-based face recognition under varying pose", Pattern Analysis and Machine Intelligence, IEEE Transactions on Volume: 21 Issue: 10, Oct. 1999, Page(s): 1081 1088.

[11] Shehu, Visar, and Agni Dika. "Using real time computer vision algorithms in automatic attendance management systems." Information Technology Interfaces (ITI), 2010 32nd International Conference on. IEEE, 2010. 